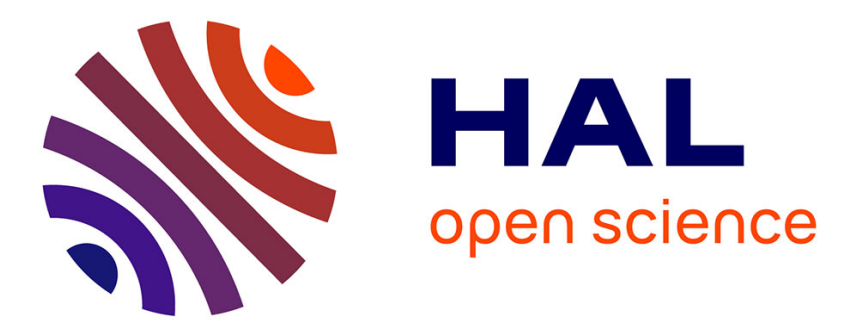

\title{
High gain observer design for nonlinear systems with time varying delayed measurements
}

\author{
Vincent van Assche, Tarek Ahmed-Ali, Hann Cheikh, Françoise
}

Lamnabhi-Lagarrigue

\section{- To cite this version:}

Vincent van Assche, Tarek Ahmed-Ali, Hann Cheikh, Françoise Lamnabhi-Lagarrigue. High gain observer design for nonlinear systems with time varying delayed measurements. 18th IFAC World Congress, Aug 2011, Milan, Italy. pp.692-696, 10.3182/20110828-6-IT-1002.02421 . hal-00648634

\section{HAL Id: hal-00648634 \\ https://hal-centralesupelec.archives-ouvertes.fr/hal-00648634}

Submitted on 6 Jul 2021

HAL is a multi-disciplinary open access archive for the deposit and dissemination of scientific research documents, whether they are published or not. The documents may come from teaching and research institutions in France or abroad, or from public or private research centers.
L'archive ouverte pluridisciplinaire HAL, est destinée au dépôt et à la diffusion de documents scientifiques de niveau recherche, publiés ou non, émanant des établissements d'enseignement et de recherche français ou étrangers, des laboratoires publics ou privés.

\section{(c)(1)}

Distributed under a Creative Commons Attribution| 4.0 International License 


\title{
High gain observer design for nonlinear systems with time varying delayed measurements
}

\author{
V. Van Assche* T. Ahmed-Ali* C. A. B. Hann* \\ F. Lamnabhi-Lagarrigue ${ }^{* *}$ \\ * GREYC UMR CNRS 6072, 6 Boulevard du Marechal Juin \\ 14050 Caen Cedex, (e-mail: vincent.vanassche@greyc.ensicaen.fr). \\ ** CNRS, Laboratoire des Signaux et Systemes (INSIS - INS2I) \\ European Embedded Control Institute (EECI) SUPELEC 3 rue Joliot \\ Curie 91192 Gif-sur-Yvette, France, (e-mail: lamnabhi@lss.supelec.fr)
}

\begin{abstract}
This work presents a simple observer for triangular nonlinear systems with time varying delayed output measurement. A sufficient condition ensuring the asymptotic convergence of the observation error towards zero is given, as well as an explicit relation between the bound of the delay and the parameter of the observer. This result is illustrated by some simulations.
\end{abstract}

Keywords: Time-varying delay systems, sampled-data systems, high gain observer.

\section{INTRODUCTION}

This paper deals with the design of nonlinear observers in the presence of delayed output measurement. This problem is particulary challenging since time delays affecting output measurements arise in a variety of applications. For example, we can cite the systems which are controlled by a remote controller through a communication system as networked control systems, or when the measurements are intrinsically delayed as for example, in biochemical reactors. In the linear case, this problem has been solved by the well-known Smith predictor Smith (1957) and several predictive control algorithms Clarke et al. (1987), Shaked and Yaesh (1998). Recently, a fundamental result has been derived for a class of nonlinear uniformly observable systems in Germani et al. (2002) with a constant delay. The authors presented a new kind of chained observers which reconstruct the states at different delayed time instants. The observer design is done step by step. At each step, the corresponding observer needs the states of all previous observers to converge. The authors showed, by using Gronwall lemma, that under some conditions on the delay, asymptotic convergence of these observers is ensured. These conditions have been relaxed in Kazantzis and Wright (2005) for a particular class of nonlinear systems. A change of coordinates based on a first-order singular partial differential equation is used, which puts the considered systems in output injection form with a stable linear part. On the other hand, in Besançon et al. (2007) a predictor for linear and nonlinear systems with delayed input has been designed. Sufficient conditions based on linear matrix inequalities are derived to guarantee the asymptotic convergence of this predictor when the delay is constant. This predictor has been extended in Ahmed-Ali et al. (2009) to triangular systems by using the framework of high gain observer. On the other hand, the case of nonlinear systems with time varying delayed measurements has been recently treated in Cacace et al. (2010). A nonlinear observer has been given for drift nonlinear systems. By using a Razumikhin approach, the authors derive some conditions ensuring the asymptotic convergence of the observation error. In the present work, we propose a nonlinear observer for nonlinear uniformly observable systems affected by a time-varying delay in the output measurements. We will see that the general high gain observer framework developed in Bornard and Hammouri (1991), Gauthier et al. (1992), to mention a few, for delay-free output measurements can be extended to systems with time varying observation delay. More precisely, if we suppose that the delay function is bounded and piecewise continuous, and without any information on its derivative, then by using a suitable Lyapunov-Krasovskii functional Fridman et al. (2008), we provide simple conditions on the upper bound of the delay which ensure asymptotic convergence of our observer. The present paper is organized as follows: In section 2, we present the class of considered systems and the different assumptions. In the third one, we detail the proposed observer and prove its convergence. In the last one, we illustrate our result through simulations on an academic example.

\section{PRELIMINARIES AND NOTATIONS}

First some mathematical notations which will be used throughout the paper are introduced.

- The euclidian norm on $\mathbb{R}^{n}$ will be denoted by $\|$.$\| .$

- The matrix $X^{T}$ represents the transposed matrix of $X$.

- $\lambda_{\min }(S)$ and $\lambda_{\max }(S)$ are the minimum and maximum eigenvalues of the square matrix $S$.

- The set of natural numbers is represented by $\mathbf{N}$.

In this paper, we consider the following class of nonlinear systems: 


$$
\begin{aligned}
& \dot{x}=A x+\phi(x, u) \\
& y=C x(t-\tau(t))
\end{aligned}
$$

where

$$
\begin{gathered}
A=\left(\begin{array}{ccccc}
0 & 1 & 0 & \ldots & 0 \\
0 & 0 & 1 & 0 & \vdots \\
\vdots & 0 & \ldots & 1 & 0 \\
\vdots & \vdots & \ldots & \ldots & 1 \\
0 & \ldots & \ldots & \ldots & 0
\end{array}\right) \\
C=\left(\begin{array}{lllll}
1 & 0 & \ldots & 0
\end{array}\right)
\end{gathered}
$$

and

$$
\phi(x, u)=\left(\begin{array}{c}
\phi_{1}(x, u) \\
\vdots \\
\phi_{n}(x, u)
\end{array}\right)
$$

The term $\tau(t)$ represents the measurement time delay, $x(t)=\left(x_{1}, \ldots, x_{k+1}, \ldots, x_{n}\right)^{T} \in \mathbb{R}^{n}$ is the vector state which is supposed unavailable. The output $y(t) \in \mathbb{R}$ is a linear function of the state $x$ at time $t-\tau(t)$. The input $u \in$ $U$ where $U$ is a compact set in $\mathbb{R}$. The functions $\phi_{i}, \quad i=$ $1, \ldots, n$ are supposed smooth. This class represents the class of uniformly observable systems. It has been shown that these models concern a wide variety of systems, such as bioreactors Bornard and Hammouri (1991), Gauthier et al. (1992). We assume that the following hypotheses are satisfied:

- Hypothesis 1. The functions $\phi_{i}(x, u)$ are triangular in $x$, i.e

$$
\frac{\partial \phi_{i}(x, u)}{\partial x^{k+1}}=0 \quad k=i, \ldots, n-1
$$

- Hypothesis 2. The functions $\phi_{i}(x, u)$ are globally Lipschitz with respect to $x$, uniformly in $u$, i.e: $\exists \beta>0$ such that $\forall\left(x, x^{\prime}\right) \in \mathbf{R}^{\mathbf{n}} \times \mathbf{R}^{\mathbf{n}}, \forall u \in U$

$$
\left|\phi_{i}(x, u)-\phi_{i}\left(x^{\prime}, u\right)\right| \leq \beta\left\|x-x^{\prime}\right\|
$$

- Hypothesis 3. $\tau(t)$ is a known piecewise-continuous time delay which satisfies $0 \leq \tau(t) \leq \tau_{1}$.

Note that the functions $\phi_{i}(x, u)$ may contains linear parts and that $\dot{x}_{n}$ depends on all $x_{1}, \ldots, x_{n}$ through $\phi_{n}(x, u)$.

\section{MAIN RESULT}

In this section, we present the main result of this paper.

Theorem 1. Consider the following observer:

$$
\begin{aligned}
& \dot{\hat{x}}=A \hat{x}+\phi(\hat{x}, u)-\theta \Delta^{-1} S^{-1} C^{T}(C \hat{x}(t-\tau(t))-y) \\
& \hat{y}=C \hat{x}(t)
\end{aligned}
$$

where $\theta$ is a positive constant satisfying $\theta>1, S$ is a symmetric positive definite matrix, which is a solution of the following equation:

$$
S A+A^{T} S-C^{T} C=-S .
$$

and $\Delta$ is a diagonal matrix which has the following form:

$$
\Delta=\operatorname{Diag}\left(1, \ldots, \frac{1}{\theta^{i-1}}, \ldots, \frac{1}{\theta^{n-1}}\right) .
$$

Then for sufficiently large positive $\theta$, there exists a positive constant $\tau_{1}$ such that $\forall \tau(t) \in\left[0, \tau_{1}\right]$ and for any initial condition, $\lim _{t \rightarrow+\infty}\|x-\hat{x}\|=0$.
Proof. First let us note the observation error $\tilde{x}=\hat{x}-x$. Then we will have:

$$
\dot{\tilde{x}}=A \tilde{x}+\phi(\hat{x}, u)-\phi(x, u)-\theta \Delta^{-1} S^{-1} C^{T} C \tilde{x}(t-\tau(t))
$$

If we apply the relation

$$
\tilde{x}(t)=\tilde{x}(t-\tau(t))+\int_{t-\tau(t)}^{t} \dot{\tilde{x}}(s) d s
$$

and the change of coordinates $\bar{x}=\Delta \tilde{x}$, system (10) can be rewritten in the following manner:

$$
\begin{aligned}
\dot{\bar{x}} & =\theta\left(A-S^{-1} C^{T} C\right) \bar{x}+\Delta(\phi(\hat{x}, u)-\phi(x, u)) \\
& +\theta S^{-1} C^{T} C \int_{t-\tau(t)}^{t} \dot{\bar{x}}(s) d s
\end{aligned}
$$

since $C \Delta=C \Delta^{-1}=C$.

In order to derive an upper bound $\tau_{1}$ for the delay $\tau(t)$, to ensure the asymptotic convergence to zero of the error $\bar{x}$, we use the following Lyapunov-Krasovskii functional from Fridman et al. (2008):

$$
W=\bar{x}^{T} S \bar{x}+\int_{t-\tau_{1}}^{t} \int_{s}^{t}\|\dot{\bar{x}}(\xi)\|^{2} d \xi d s .
$$

This functional can be written after some manipulations as follows:

$$
W=\bar{x}^{T} S \bar{x}+\int_{t-\tau_{1}}^{t}\left(s-t+\tau_{1}\right)\|\dot{\bar{x}}(s)\|^{2} d s .
$$

If we compute its time derivative, we obtain

$$
\begin{aligned}
\dot{W} & \leq \theta \bar{x}^{T}\left(A^{T} S+S A-2 C^{T} C\right) \bar{x} \\
& +2 \bar{x}^{T} S \Delta(\phi(\hat{x}, u)-\phi(x, u)) \\
& +2 \theta \bar{x}^{T} C^{T} C \int_{t-\tau(t)}^{t} \dot{\bar{x}}(s) d s+\tau_{1}\|\dot{\bar{x}}(t)\|^{2} \\
& -\int_{t-\tau_{1}}^{t}\|\dot{\bar{x}}(s)\|^{2} d s .
\end{aligned}
$$

Using (8), we have

$$
\begin{aligned}
\dot{W} & \leq-\theta \bar{x}^{T} S \bar{x}+2 \bar{x}^{T} S \Delta(\phi(\hat{x}, u)-\phi(x, u)) \\
& -\theta \bar{x}^{T} C^{T} C \bar{x}+2 \theta \bar{x}^{T} C^{T} C \int_{t-\tau(t)}^{t} \dot{\bar{x}}(s) d s \\
& +\tau_{1}\|\dot{\bar{x}}(t)\|^{2}-\int_{t-\tau_{1}}^{t}\|\dot{\bar{x}}(s)\|^{2} d s .
\end{aligned}
$$

Using the triangular structure and the Lipschitz properties of the functions $\phi_{i}$, we can easily derive that if $\theta>1$, then

$$
\|\Delta(\phi(\hat{x}, u)-\phi(x, u))\| \leq \sqrt{n} \beta\|\bar{x}\| .
$$

From this, we deduce that there exists a constant

$$
k_{1}=2 \frac{\lambda_{\max }(S)}{\lambda_{\min }(S)} \sqrt{n} \beta
$$

such that

$$
\left\|2 \bar{x}^{T} S \Delta(\phi(\hat{x}, u)-\phi(x, u))\right\| \leq k_{1} V
$$

with $V=\bar{x}^{T} S \bar{x}$

Using the following property: 


$$
\begin{aligned}
2 \theta \bar{x}^{T} C^{T} C \int_{t-\tau(t)}^{t} \dot{\bar{x}}(s) d s-\theta \bar{x}^{T} C^{T} C \bar{x} \\
=-\theta\left(C x-C \int_{t-\tau(t)}^{t} \dot{\bar{x}}(s) d s\right)^{T} \\
\\
\cdot\left(C x-C \int_{t-\tau(t)}^{t} \dot{\bar{x}}(s) d s\right) \\
+\theta\left(\int_{t-\tau(t)}^{t} \dot{\bar{x}}(s) d s\right)^{T} C^{T} C\left(\int_{t-\tau(t)}^{t} \dot{\bar{x}}(s) d s\right),
\end{aligned}
$$

one gets

$$
\begin{aligned}
2 \theta \bar{x}^{T} C^{T} C \int_{t-\tau(t)}^{t} \dot{\bar{x}}(s) d s-\theta \bar{x}^{T} C^{T} C \bar{x} \\
\leq \theta\left(\int_{t-\tau(t)}^{t} \dot{\bar{x}}(s) d s\right)^{T} C^{T} C\left(\int_{t-\tau(t)}^{t} \dot{\bar{x}}(s) d s\right) .
\end{aligned}
$$

From this, we will have

$$
\begin{aligned}
\dot{W} & \leq-\theta V+k_{1} V \\
& +\theta\left(\int_{t-\tau(t)}^{t} \dot{\bar{x}}(s) d s\right)^{T} C^{T} C\left(\int_{t-\tau(t)}^{t} \dot{\bar{x}}(s) d s\right) \\
& +\tau_{1}\|\dot{\bar{x}}(t)\|^{2}-\int_{t-\tau_{1}}^{t}\|\dot{\bar{x}}(s)\|^{2} d s .
\end{aligned}
$$

Using equation (12), one can write

$$
\begin{array}{r}
\|\dot{\bar{x}}(t)\| \leq \theta \alpha\|\bar{x}\|+\|\Delta(\phi(\hat{x}, u)-\phi(x, u))\| \\
+\theta \lambda_{\max }\left(S^{-1}\right)\|I\|,
\end{array}
$$

where

$$
\begin{aligned}
& \alpha=\left\|A-S^{-1} C^{T} C\right\|, \\
& I=\int_{t-\tau(t)}^{t} \dot{\bar{x}}(s) d s .
\end{aligned}
$$

If $\theta>1$. Since, as proven by Gauthier et al. (1992),

one has

$$
\|\Delta(\phi(\hat{x}, u)-\phi(x, u))\| \leq \sqrt{n} \beta\|\bar{x}\|,
$$

$$
\|\dot{\bar{x}}(t)\| \leq \theta[\alpha+\sqrt{n} \beta]\|\bar{x}\|+\theta \lambda_{\max }\left(S^{-1}\right)\|I\| .
$$

Using the Young inequality, one can write

$$
\|\dot{\bar{x}}(t)\|^{2} \leq \theta^{2} k_{2}\left[V+\|I\|^{2}\right]
$$

where

$$
k_{2}=2 \sup \left\{\left(\frac{\alpha+\sqrt{n} \beta}{\lambda_{\min }(S)}\right)^{2}, \lambda_{\max }^{2}\left(S^{-1}\right), 1\right\} .
$$

Using this and equation (15), we will have:

$$
\begin{aligned}
\dot{W} \leq-\theta V+ & k_{1} V+\theta I^{T} C^{T} C I \\
& +\tau_{1} \theta^{2} k_{2}\left[V+\|I\|^{2}\right]-\int_{t-\tau_{1}}^{t}\|\dot{\bar{x}}(s)\|^{2} d s
\end{aligned}
$$

If we use the following Jensen's inequality:

$$
\|I\|^{2} \leq \tau_{1} \int_{t-\tau_{1}}^{t}\|\dot{\bar{x}}(s)\|^{2} d s
$$

$$
\begin{aligned}
\dot{W} \leq-(\theta- & \left.k_{1}-\tau_{1} \theta^{2} k_{2}\right) V \\
& \quad-\left(1-\theta \tau_{1}-\tau_{1}^{2} \theta^{2} k_{2}\right) \int_{t-\tau_{1}}^{t}\|\dot{\bar{x}}(s)\|^{2} d s .
\end{aligned}
$$

Let us choose $\tau_{1}=\frac{1}{2 k_{2} \theta}$. Then (26) becomes

$$
\dot{W} \leq-\left(\frac{\theta}{2}-k_{1}\right) V-\left(1-\frac{3}{8 k_{2}}\right) \int_{t-\tau_{1}}^{t}\|\dot{\bar{x}}(s)\|^{2} d s
$$

Since $k_{2}>1$ and $\theta>1$, then $\left(1-\frac{3}{8 k_{2}}\right)>0$. From this we can say that the inequality

$$
\dot{W}<-\left(\frac{\theta}{2}-k_{1}\right) V \leq 0
$$

fulfilled under the following conditions;

$$
\begin{aligned}
\theta & >\sup \left\{1,2 k_{1}\right\} \\
\tau(t) & \in\left[0, \frac{1}{2 k_{2} \theta}\right]
\end{aligned}
$$

Integrating (28) and using Barbalat's lemma, we conclude that

$$
\lim _{t \rightarrow+\infty}\|\tilde{x}(t)\|=0 .
$$

To summarize Theorem 1, it gives an estimation of the maximum delay supported by observer (7) which enables $\hat{x}(t) \rightarrow x(t)$, once $\theta$ has been fixed according to the first inequality of conditions (29).

\subsection{Application to sampled-data case}

The result stated in Theorem 1, can be easily extended to the case of sampled measurements. Let the monotonically increasing sequence $t_{k}, k \in \mathbf{N}$ with $\lim _{k \rightarrow+\infty} t_{k}=+\infty$ which represents the sampling instants. The maximum sampling time interval is noted $T_{e}=\max _{k \in \mathbf{N}}\left(t_{k+1}-t_{k}\right)$. Corollary 1. Let us consider the following observer:

$$
\begin{aligned}
\dot{\hat{x}} & =A \hat{x}+\phi(\hat{x}, u) \\
& -\theta \Delta^{-1} S^{-1} C^{T}\left(C \hat{x}\left(t_{k}\right)-y\left(t_{k}\right)\right) \quad \forall t \in\left[t_{k}, t_{k+1}\right) \\
\hat{y} & =C \hat{x}(t)
\end{aligned}
$$

and suppose that the following conditions are fulfilled

$$
\begin{aligned}
\theta & >\sup \left\{1,2 k_{1}\right\} \\
T_{e} & \leq \frac{1}{2 k_{2} \theta}
\end{aligned}
$$

then (30) is a global asymptotic observer for systems (1).

The proof of this corollary is obliviously derived from fact $\forall t \in\left[t_{k}, t_{k+1}\right)$ we have $t_{k}=t-\tau(t)$, with $\tau(t)=t-t_{k}$ and $\dot{\tau}(t)=1$. This properties allow us to use the result of Theorem 1.

\section{EXAMPLES}

\subsection{Simulation with random varying delay}

In order to illustrate the result presented above, consider the following system:

$$
\left\{\begin{aligned}
\dot{x}_{1}(t) & =c_{1} x_{2}(t)-l_{1} x_{1}(t), \\
\dot{x}_{2}(t) & =c_{2} \sin \left(x_{2}(t)\right)+c_{3} \cos \left(x_{2}(t)\right)+c_{4} u(t), \\
y(t) & =x_{1}(t-\tau(t)) .
\end{aligned}\right.
$$


This system can be written in the form (1) with the following change of variable:

$$
\begin{aligned}
& z_{1}(t)=x_{1}(t), \\
& z_{2}(t)=c_{1} x_{2}(t),
\end{aligned}
$$

which leads to

$$
\left\{\begin{array}{l}
\dot{z}(t)=A z(t)+\phi(z(t), u(t)) \\
y(t)=z_{1}(t-\tau(t))
\end{array}\right.
$$

where

$$
A=\left(\begin{array}{ll}
0 & 1 \\
0 & 0
\end{array}\right) \quad \phi(z(t), u(t))=\left(\begin{array}{c}
\phi_{1}(z(t)) \\
\left.\phi_{2}(z(t), u)\right)
\end{array}\right)
$$

with

$$
\begin{aligned}
\phi_{1}(z(t))= & -l_{1} z_{1}(t) \\
\phi_{2}(z(t), u(t))= & c_{1} c_{2} \sin \left(z_{2}(t) / c_{1}\right) \\
& +c_{1} c_{3} \cos \left(z_{2}(t) / c_{1}\right) \\
& +c_{1} c_{4} u(t) .
\end{aligned}
$$

The function $\tau(t)$ is piecewise constant with values in the range $\left[0, \tau_{1}\right]$. In the simulations below, the value of $\tau(t)$ is set randomly at regular time interval $\delta_{1}=0.1$.

The values of the system parameters are set to $c_{1}=1$, $c_{2}=0.02, c_{3}=0.02, c_{4}=8, l_{1}=l_{2}=0.04$, the initial conditions for the system are, $\forall t \in\left[-\tau_{1} 0\right], x_{1}(t)=x_{2}(t)=$ 50. The input function is $u(t)=\sin (0.35 t)$ for $t \geq 0$.

The solution of (8) for this system is

$$
S=\left(\begin{array}{cc}
1 & -1 \\
-1 & 2
\end{array}\right)
$$

with $\lambda_{\min }(S)=0.382, \lambda_{\max }(S)=2.618$. One has $\beta=$ $0.04,(18)$ leads to $k_{1}=0.7755$, and (24) leads to $k_{2}=$ 31.5377. The observer parameter is set to the smallest value allowed by $(29 \mathrm{a}): \theta=1.55$. The maximum value for the delay is then given by $(29 \mathrm{~b}): \tau_{1}=10^{-2}$. The initial conditions for the observer are, $\forall t \in\left[\begin{array}{ll}-\tau_{1} & 0\end{array}\right]$, $\hat{x}_{1}(t)=\hat{x}_{2}(t)=0$. As we can see, the simulations displayed in Figure 1 show clearly the asymptotic convergence of the observation error.

\subsection{Simulation with sampled output}

The simulations displayed in Figure 3 show also the exponential convergence of observer (30) in the case of a sampled output with sample period 0.01 .

\section{CONCLUSION}

In this paper, a high gain observer with time varying delayed measurements has been presented. An explicit relation between the gain $\theta$ of the considered observer and the upper bound of the delay is given. This condition ensures asymptotic convergence of the observation error. As we can see, this bound can be very small for high values of $\theta$. This means that for relatively important delays, the considered observer can not guarantee an asymptotic convergence. To overcome this problem, we will present in a future work a cascade of high gain observers based on the delay bound computed here.
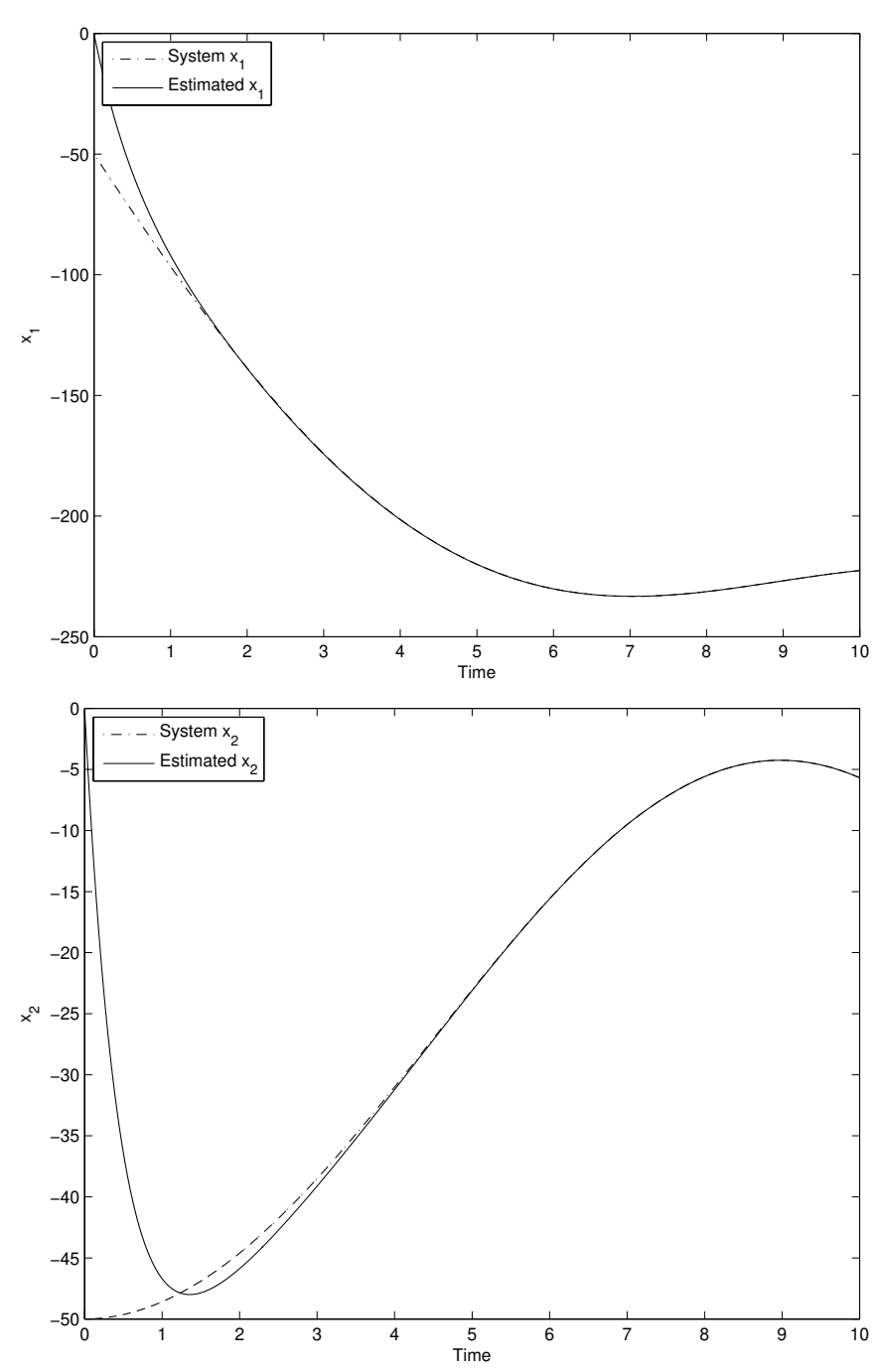

Fig. 1. Evolution of the state variables and their estimate, random delay with $\tau_{1}=0.01$.

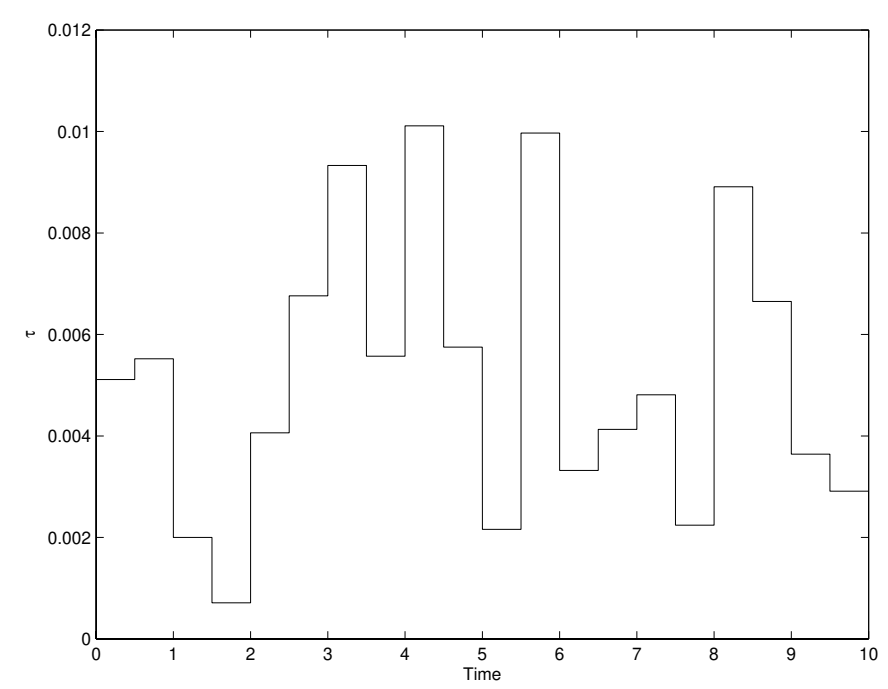

Fig. 2. Evolution of the random delay $\tau(t)$ with $\tau_{1}=0.01$. 

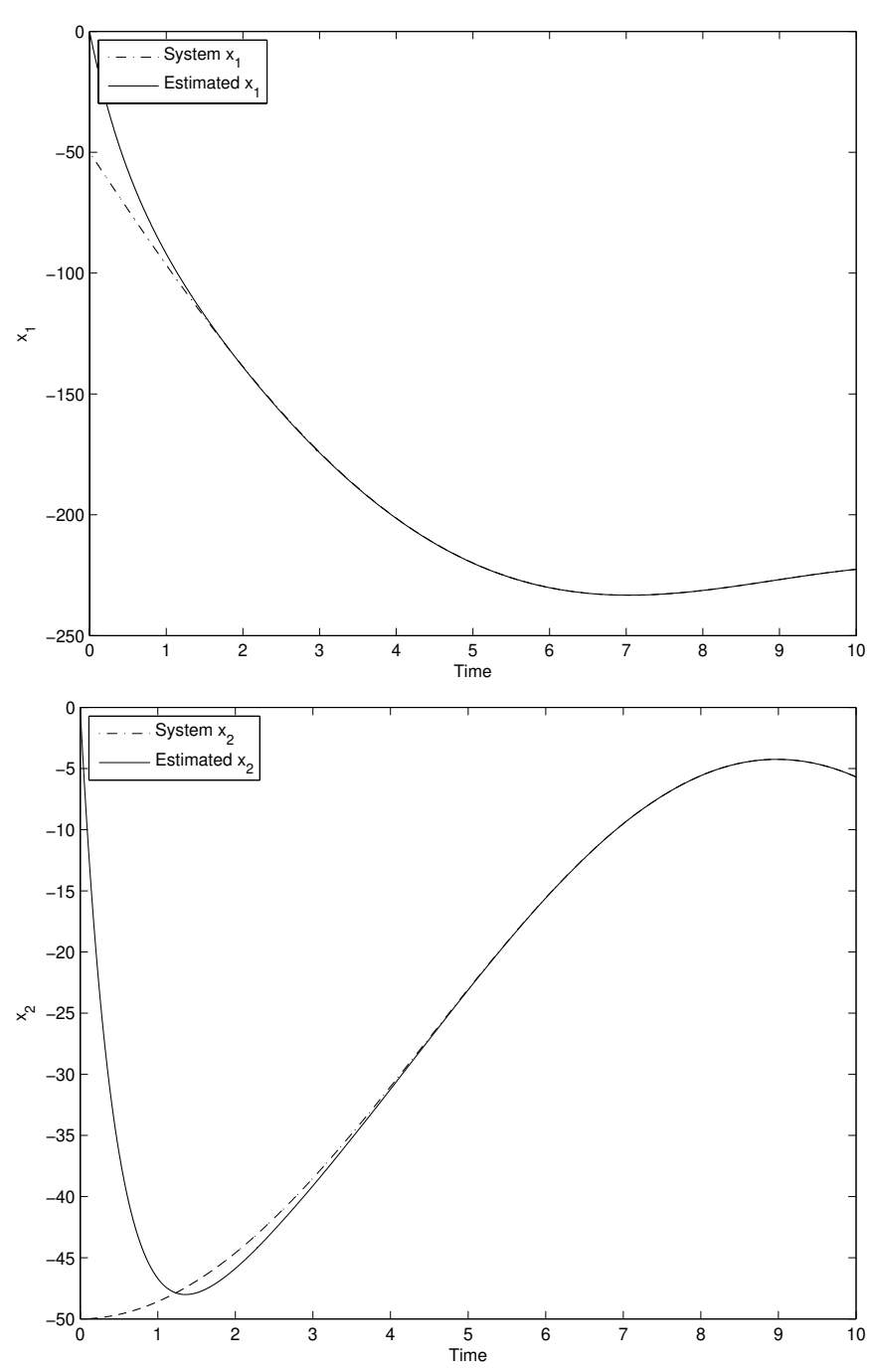

Fig. 3. Evolution of the state variables and their estimate in the case of a sampled output with sample period 0.01

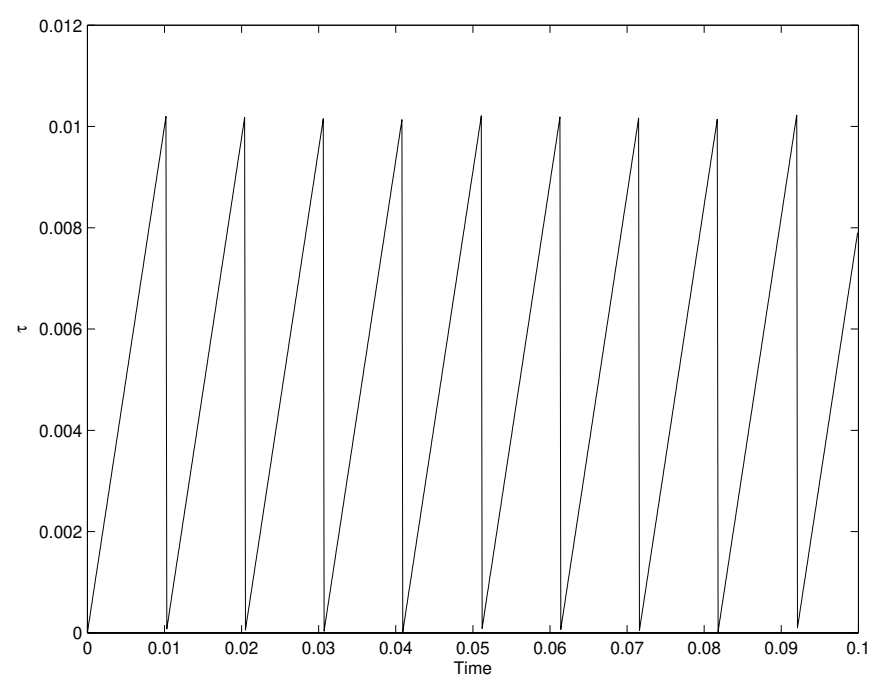

Fig. 4. Evolution of the delay $\tau(t)=t-t_{k}, t \in\left[t_{k}, t_{k+1}\right)$ $k \in \mathbf{N}$ in the case of a sampled output with sample period 0.01

\section{REFERENCES}

T. Ahmed-Ali, E. Cherrier, and M.MSaad. Cascade high gain observers for nonlinear systems with delayed output. In 48th IEEE-CDC, Shanghai, pages 8226 8231, 2009.

G. Besançon, D. Georges, and Z. Benayache. Asymptotic state prediction for continuous-time systems with delayed input and application to control. In Proceedings of the European Control Conference, Kos, Greece, 2007.

G. Bornard and H. Hammouri. A high gain observer for a class of uniformly observable systems. In Proceedings of the 30th IEEE Conference on Decision and Control, Brighton, England, 1991.

F. Cacace, A. Germani, and C. Manes. An observer for a class of nonlinear systems with time varying observation delay. Sys. \& Control Letters, 59:305-312, 2010.

D.W. Clarke, C. Mohtadi, and P.S. Tuffs. Generalized predictive control-part I and II. Automatica, 23:137160, 1987.

E. Fridman, M. Dambrine, and N. Yeganefar. On inputto-state stability of systems with time-delay: A matrix inequalities approach. Automatica, 44:2364-2369, 2008.

J.P. Gauthier, H. Hammouri, and S. Othman. A simple obsever for nonlinear systems: Application to bioreactors. IEEE Trans. Automatic Control, 37(6):875-880, 1992.

A. Germani, C. Manes, and P. Pepe. A new approach to state observation of nonlinear systems with delayed output. IEEE Trans. Automatic Control, 47(1):96-101, 2002.

N. Kazantzis and R.A. Wright. Nonlinear observer design in the presence of delayed output measurements. Sys. E Control Letters, 54:877-886, 2005.

U. Shaked and I. Yaesh. H-infinity static output-feedback control of linear continuous-time systems with delay. IEEE Trans. Automatic Control, 43:1431-1436, 1998.

O.J.M. Smith. Closer control of loops with dead-time. Chem. Eng. Prog., 53(5):217-219, 1957. 